\title{
Serum ferritin levels are associated with advanced liver fibrosis in treatment-naive autoimmune hepatitis
}

\author{
Qingling Chen ${ }^{1,2+}$, Min Gao ${ }^{2 \dagger}$, Hang Yang ${ }^{1,2}$, Ling Mei ${ }^{1,2}$, Rui Zhong ${ }^{3}$, Ping Han ${ }^{2}$, Peiyan Liu ${ }^{1,2}$, Lili Zhao ${ }^{2}$, \\ Jing Wang ${ }^{2}$ and Jia Li ${ }^{2^{*}}$
}

\begin{abstract}
Background and aim: The association between iron-metabolism-related variables and liver fibrosis in chronic hepatitis $C$ and nonalcoholic fatty liver disease is now well known. However, the relationship has not been extensively studied in autoimmune hepatitis (AlH). We aimed to investigate the association between variables associated with iron metabolism and advanced liver fibrosis among untreated patients with $\mathrm{AlH}$.

Methods: Ninety-seven untreated AlH patients were enrolled in this cross-sectional study. All participants underwent iron metabolism index detection and liver biopsy. Multiple logistic regression analysis was used to explore the association of iron-metabolism-related variables with advanced liver fibrosis.

Results: Among the 97 AlH patients, 38 (39.2\%) had advanced liver fibrosis, and 59 (60.8\%) did not. In multivariate logistic regression analysis, immunoglobulin G (odds ratio [OR], 1.123; 95\% confidence interval [CI] 1.023-1.232, $P=0.014)$, platelet count $(\mathrm{OR} 0.988 ; 95 \% \mathrm{Cl} 0.979-0.997, P=0.013)$, prothrombin time (OR $1.758 ; 95 \% \mathrm{Cl} 1.143-2.704$, $P=0.010)$ and ferritin (OR 1.002; $95 \% \mathrm{Cl} 1.001-1.004, P=0.012)$ were independent risk factors for predicting advanced liver fibrosis in AlH patients.
\end{abstract}

Conclusion: Higher serum ferritin was independently associated with advanced liver fibrosis among patients with treatment-naive AlH.

Keywords: Autoimmune hepatitis, Ferritin, Iron metabolism disorders, Liver fibrosis

\section{Introduction}

Autoimmune hepatitis (AIH) is a chronic and progressive inflammatory liver disease characterized by elevated levels of serum aminotransferases and immunoglobulin G (IgG), the presence of autoantibodies, and interface hepatitis with lymphoplasmacytic infiltration in liver histology [1]. AIH is a heterogeneous condition that occurs globally in children and adults of

\footnotetext{
*Correspondence: 18622663700@163.com

${ }^{\dagger}$ Qingling Chen and Min Gao have contributed equally to this work

2 Department of Hepatology, Tianjin Second People's Hospital, No. 7, Sudi

South Road, Nankai District, Tianjin 300192, China

Full list of author information is available at the end of the article
}

all ages and affects all ethnicities, with a female predominance. The prevalence of AIH is significantly different among different geographical regions and ethnic populations, and it is higher in North America and Europe than in the Asia-Pacific area [2-4]. Although previous AIH studies were mostly conducted in Europe and North America, an increasing number of studies from eastern countries have been reported in the past 10 years [3]. Due to the lack of specific diagnostic markers in AIH patients, most patients have significant fibrosis or even cirrhosis at the initial presentation [5]. An evaluation of the liver fibrosis stages is essential for the selection of treatment strategies 
and the estimation of the long-term prognosis for $\mathrm{AIH}$ patients.

Liver biopsy is considered the gold standard for the assessment of liver fibrosis in AIH patients [6, 7]. However, the clinical application of liver biopsy is often limited by its invasiveness, high cost, sampling errors, risk of complications, and poor patient compliance, particularly in the follow-up period $[8,9]$. Therefore, it is important to develop noninvasive and convenient markers capable of accurately evaluating the stages of fibrosis in AIH patients. Indeed, several noninvasive markers for assessing liver fibrosis and cirrhosis have been applied in clinical practice, including the fibrosis index based on 4 factors (FIB-4), the aspartate transaminase-to-platelet ratio index (APRI), the aspartate aminotransferase/alanine aminotransferase ratio (AAR), and the AAR/platelet ratio index (AARPRI) [10,11]. These noninvasive markers can be used to detect cirrhosis in patients with chronic viral hepatitis and nonalcoholic fatty liver disease. However, the ability of these markers to detect early liver fibrosis and cirrhosis in AIH patients remains uncertain. In recent years, liver stiffness measurement (LSM) has been widely investigated as a noninvasive method to quantify liver fibrosis, especially in metabolic and viral liver diseases [12, 13]. Nevertheless, the application of LSM has not been widely investigated in patients with AIH. Hartl et al. [14] demonstrated that transient elastography is a reliable tool for the detection of liver fibrosis in treated patients with $\mathrm{AIH}$, but it showed limited value in therapy-naive individuals with active hepatitis.

Disturbances in iron regulation are reported in diverse chronic liver diseases beyond haemochromatosis and are mostly associated with a worse disease course [15-22]. Hyperferritinaemia has been the main manifestation of disturbed iron homeostasis in chronic liver diseases [16-19]. Elevated serum ferritin levels were reported to be independently associated with advanced liver fibrosis in patients with chronic hepatitis $C$ and nonalcoholic fatty liver disease [23-27]. However, to date, the relationship between iron dysregulation and liver fibrosis has not been extensively studied in patients with AIH. Therefore, we analysed the status of the iron-metabolism-related variables and compared them with fibrosis stages in liver biopsy specimens in a cohort of untreated AIH patients. Nevertheless, there is usually increased inflammation in the liver with acute phase reaction at diagnosis, and ferritin levels can reflect inflammation; therefore, we will pay more attention to these same variables in treated patients after remission, which could valuable for estimations of disease progression, in future research.

\section{Methods}

\section{Study population}

In this retrospective study, we reviewed the medical records of adult AIH patients who underwent liver biopsy prior to immunosuppressive therapy between March 2013 and March 2021 in the Department of Hepatology, Tianjin Second People's Hospital, Tianjin, China. The AIH diagnosis was made if patients met the criteria of (1) a 1999 revised International Autoimmune Hepatitis Group (IAIHG) score $\geq 10$ and/or (2) an 2008 IAIHG simplified AIH score $\geq 6[28,29]$. Patients with primary biliary cholangitis, primary sclerosing cholangitis, autoimmune overlap syndromes, viral hepatitis, alcoholic or nonalcoholic fatty liver disease, drug-induced liver disease, hereditary metabolic liver disease, hereditary haemochromatosis, hepatocellular carcinoma, and severe systemic diseases were excluded. Finally, a total of $97 \mathrm{AIH}$ patients were included in this cross-sectional analysis.

This study protocol was approved by the Ethics Committee of Tianjin Second People's Hospital and conducted according to the principles of the Declaration of Helsinki. We obtained written informed consent from all patients.

\section{General characteristics and laboratory test results}

The medical records of all enrolled patients diagnosed with AIH were reviewed. Demographic data (age and sex) and laboratory results (of routine blood tests, biochemistry tests, and immunology tests) were collected and analysed. For all patients, blood samples were collected on the same day within 1 week before liver biopsy. Serum alanine aminotransferase (ALT), aspartate aminotransferase (AST), alkaline phosphatase (ALP), gamma-glutamyl transpeptidase (GGT), total bilirubin (TBIL), albumin (ALB), and IgG were detected by a Hitachi 7180 Automatic Biochemical Analyser (Hitachi, Ltd, Tokyo, Japan). The cut-off values for each parameter were set as values above the upper limit of the normal range, i.e., at $50 \mathrm{U} / \mathrm{L}$ for ALT, $40 \mathrm{U} / \mathrm{L}$ for AST, $125 \mathrm{U} / \mathrm{L}$ for ALP, $60 \mathrm{U} / \mathrm{L}$ for GGT, $20.52 \mu \mathrm{mol} / \mathrm{L}$ for TBIL, $55 \mathrm{~g} / \mathrm{L}$ for ALB, and $16 \mathrm{~g} / \mathrm{L}$ for IgG. Antibodies were assayed by indirect immunofluorescence or immunoblotting using a CycleBlot 48 automatic Western blotting instrument, and the starting dilution was 1:100. The complete blood count was measured using a Sysmex XN-2000 haematology analyser (Sysmex corporation, Kobe, Japan) according to the manufacturer's recommendation. The coagulation tests were performed by the clotting method on the automatic coagulometer "STAGO Compact" ("Diagnostica Stago", France).

All the patients were assayed for variables related to iron metabolism: serum iron (colorimetric test), transferrin (immunoturbidimetry), ferritin (latex-enhanced immunoturbidimetric method), and unsaturated iron 
binding capacity (UIBC) (colorimetric test) following standard laboratory procedures. Subsequently, total iron binding capacity (TIBC) was calculated as UIBC + iron, and the transferrin saturation index (TSI) was calculated as serum iron/TIBC $\times 100$.

\section{Histological assessments}

Ultrasound-guided liver biopsy was performed using a 16-gauge disposable needle for all AIH patients under local anaesthesia. The liver pathology diagnosis required a liver specimen of at least $1.0 \mathrm{~cm}$, which contains a minimum of 10 portal tracts. Each obtained specimen was fixed in $10 \%$ neutral-buffered formalin, embedded in paraffin, and routinely stained with haematoxylin-eosin and Masson's trichrome. Liver tissue specimens were independently evaluated by two experienced liver pathologists blinded to patient clinical characteristics. Typical features of AIH include interface hepatitis, lymphocytic/ lymphoplasmocytic infiltration in portal tracts, and hepatic rosette formation [29]. The histological assessment of the inflammatory grades and the fibrosis stages were based on the Batts-Ludwig scoring system [30, 31]. Liver fibrosis was classified into the following 5 stages: F0, no fibrosis; F1, portal fibrosis without septa; F2, portal fibrosis with few septa; F3, numerous septa without cirrhosis; and F4, cirrhosis [31]. Stages F3-F4 are defined as advanced liver fibrosis.

\section{Statistical analysis}

We tested whether the continuous variables analysed showed a normal distribution using the KolmogorovSmirnov test. Normally distributed values are expressed as the mean ( \pm standard deviation), while nonnormally distributed values are expressed as the median (quartile 25 , quartile 75$)$. Data for categorical variables are presented as the number (n) and proportion (\%). Data were analysed by Spearman's coefficient of correlation (r), Student's $t$ test, the Mann-Whitney $U$ test and the chisquare test where applicable. The association between variables and advanced liver fibrosis was assessed by binary logistic regression analysis. Univariate logistic regression analysis was performed by the "Enter" method, and the variables that were statistically significant were entered into multivariate logistic regression analysis. The receiver operating characteristic (ROC) curve of ferritin was analysed to determine the best cutoff value for the prediction of advanced fibrosis stage by the DeLong method. Statistical analyses were performed using SPSS Statistics 26 (IBM, New York, NY, USA) and MedCalc Statistical Software version 15.8 (MedCalc Software bvba, Ostend, Belgium; https://www.medcalc.org; 2015). A two-sided $P<0.05$ was considered statistically significant.

\section{Results}

\section{General characteristics of the patients}

A total of 97 AIH patients with a mean age of 54.0 years were included in this study. The majority of patients were female $(84.5 \%)$, and the prevalence of autoantibodies was $85.6 \%$. The median levels of IgG, ALT, and AST were $16.80(13.35,21.10) \mathrm{g} / \mathrm{L}, 88.0(32.0,322.5) \mathrm{U} / \mathrm{L}$, and $113.0(35.0,401.0) \mathrm{U} / \mathrm{L}$, respectively. The distribution of patients according to liver fibrosis stage is as follows: F0, 11 (11.3\%) patients; F1, 22 (22.7\%) patients; F2, 26 (26.8\%) patients; F3, 21 (21.6\%) patients; and F4, 17 $(17.5 \%)$ patients. Further information on the characteristics of the patients is shown in Table 1.

\section{General characteristics of patients by liver fibrosis stage}

The included patients were divided into an advanced fibrosis group and a nonadvanced fibrosis group according to the results of liver biopsy analysis. Among the 97 AIH patients, 38 (39.2\%) had advanced liver fibrosis, and $59(60.8 \%)$ did not. No significant difference in sex proportion was observed between the two groups $(P=0.943)$, but the age of patients in the advanced fibrosis group was significantly higher than that in the nonadvanced fibrosis group $(P=0.010)$. The IgG, TBIL, prothrombin time (PT), ferritin, and TSI in the advanced fibrosis group were significantly higher than those in the nonadvanced fibrosis group $(P<0.05)$, while the ALB, platelet (PLT) count, transferrin, UIBC, and TIBC in the advanced fibrosis group were significantly lower than those in the nonadvanced fibrosis group $(P<0.05)$. Significant differences between the two groups were not observed for other parameters $(P>0.05)$, as shown in Table 2.

\section{Correlations between iron-metabolism-related variables and the clinical characteristics of patients}

The correlations between iron-metabolism-related variables and clinical characteristics are summarized in Table 3. As expected, serum iron levels were positively correlated with ferritin and TSI but negatively correlated with transferrin, UIBC, and TIBC $(P<0.05)$. Serum iron, ferritin and TSI were positively correlated with age, TBIL, and fibrosis stage $(P<0.05)$, while transferrin, UIBC, and TIBC were negatively correlated with age, TBIL, and fibrosis stage $(P<0.05)$. Serum iron, ferritin and TSI were positively correlated with ALT, AST, ALP, and GGT $(P<0.05)$, while UIBC was negatively correlated with ALT, AST, ALP, and GGT $(P<0.05)$. Serum iron levels and TSI were negatively correlated with ALB and the PLT count $(P<0.05)$, while transferrin, UIBC, and TIBC were positively correlated with 
Table 1 General characteristics of the patients with autoimmune hepatitis

\begin{tabular}{|c|c|}
\hline Variables & $n=97$ \\
\hline Age (years) & $54.0 \pm 11.3$ \\
\hline Female, n (\%) & $82(84.5)$ \\
\hline Autoantibodies positive, n (\%) & $83(85.6)$ \\
\hline ANA,$+ n(\%)$ & $78(80.4)$ \\
\hline ASMA,$+ n(\%)$ & $24(24.7)$ \\
\hline Anti-LKM1 +, n (\%) & $3(3.1)$ \\
\hline Anti-LC1, n (\%) & $2(2.1)$ \\
\hline Anti-SLA/LP, n (\%) & $2(2.1)$ \\
\hline $\lg G(g / L)$ & $16.80(13.35,21.10)$ \\
\hline $\mathrm{ALT}(\mathrm{U} / \mathrm{L})$ & $88.0(32.0,322.5)$ \\
\hline $\mathrm{AST}(\mathrm{U} / \mathrm{L})$ & $113.0(35.0,401.0)$ \\
\hline ALP (U/L) & $110.0(75.5,151.0)$ \\
\hline GGT (U/L) & $125.9(59.0,248.0)$ \\
\hline TBIL ( $\mu \mathrm{mol} / \mathrm{L})$ & $20.0(12.7,68.4)$ \\
\hline ALB $(g / L)$ & $40.1 \pm 5.6$ \\
\hline $\operatorname{PLT}\left(\times 10^{9} / \mathrm{L}\right)$ & $184.8 \pm 70.5$ \\
\hline PT (s) & $13.2 \pm 1.5$ \\
\hline Iron ( $\mu \mathrm{mol} / \mathrm{L})$ & $27.7 \pm 14.4$ \\
\hline Transferrin (g/L) & $2.67(2.28,3.17)$ \\
\hline Ferritin $(\mu \mathrm{g} / \mathrm{L})$ & $214.0(78.0,515.0)$ \\
\hline UIBC ( $\mu \mathrm{mol} / \mathrm{L})$ & $31.2(21.2,48.2)$ \\
\hline TIBC ( $\mu \mathrm{mol} / \mathrm{L})$ & $59.8(53.8,68.9)$ \\
\hline TSI (\%) & $43.6(28.2,64.2)$ \\
\hline AlH score (1999) & $15.8 \pm 2.7$ \\
\hline AlH score (2008) & $7.5 \pm 1.3$ \\
\hline \multicolumn{2}{|l|}{ Histology (inflammation), n (\%) } \\
\hline Go & $0(0)$ \\
\hline G1 & $3(3.1)$ \\
\hline G2 & $42(43.3)$ \\
\hline G3 & $40(41.2)$ \\
\hline G4 & $12(12.4)$ \\
\hline \multicolumn{2}{|l|}{ Histology (fibrosis), n (\%) } \\
\hline F0 & $11(11.3)$ \\
\hline F1 & $22(22.7)$ \\
\hline $\mathrm{F} 2$ & $26(26.8)$ \\
\hline F3 & 21 (21.6) \\
\hline F4 & $17(17.5)$ \\
\hline
\end{tabular}

Data are expressed as mean ( \pm standard deviation), median (quartile 25 , quartile 75) or number (proportion)

ANA antinuclear antibody, ASMA anti-smooth muscle antibody, Anti-LKM1 anti-liver kidney microsomal antibody type 1, Anti-LC1 anti-liver cytosol type 1 antibody, Anti-SLA/LP anti-soluble liver antigen or anti-liver-pancreas antibodies, IgG immunoglobulin G, ALT alanine aminotransferase, AST aspartate aminotransferase, $A L P$ alkaline phosphatase, GGT gamma-glutamyl transpeptidase, TBIL total bilirubin, $A L B$ albumin, $P L T$ platelet, $P T$ prothrombin time, UIBC unsaturated iron binding capacity, $T I B C$ Total iron binding capacity, $T S /$ transferrin saturation index
Table 2 General characteristics of subjects by liver fibrosis stage

\begin{tabular}{lllr}
\hline Variables & F0-2 $(\mathbf{n}=\mathbf{5 9})$ & F3-4 $(\mathbf{n}=\mathbf{3 8})$ & \multicolumn{1}{c}{$\boldsymbol{P}$} \\
\hline Age $($ years $)$ & $51.7 \pm 11.7$ & $57.5 \pm 9.7$ & 0.010 \\
Female, $n(\%)$ & $50(84.7)$ & $32(84.2)$ & 0.943 \\
Autoantibodies & $48(81.4)$ & $35(92.1)$ & 0.141 \\
positive, $n(\%)$ & & & \\
IgG $(\mathrm{g} / \mathrm{L})$ & $15.00(12.90,19.18)$ & $18.70(15.85,24.60)$ & 0.002 \\
ALT $(\mathrm{U} / \mathrm{L})$ & $79.0(29.0,387.0)$ & $122.5(39.8,311.3)$ & 0.414 \\
AST $(\mathrm{U} / \mathrm{L})$ & $69.0(30.0,387.0)$ & $173.0(45.8,421.0)$ & 0.093 \\
ALP $(\mathrm{U} / \mathrm{L})$ & $101.0(71.0,151.0)$ & $118.0(78.5,161.0)$ & 0.206 \\
GGT $(\mathrm{U} / \mathrm{L})$ & $122.0(73.0,184.0)$ & $132.0(54.3,298.5)$ & 0.444 \\
TBIL $(\mu \mathrm{mol} / \mathrm{L})$ & $17.4(11.8,33.5)$ & $28.0(14.8,134.6)$ & 0.026 \\
ALB $(\mathrm{g} / \mathrm{L})$ & $41.1 \pm 5.0$ & $38.6 \pm 6.2$ & 0.035 \\
PLT $\left(\times 10^{9} / \mathrm{L}\right)$ & $208.5 \pm 68.2$ & $148.1 \pm 57.5$ & $<0.001$ \\
PT $(\mathrm{s})$ & $12.7 \pm 1.2$ & $13.9 \pm 1.6$ & $<0.001$ \\
Iron $(\mu \mathrm{mol} / \mathrm{L})$ & $25.9 \pm 16.0$ & $30.5 \pm 11.0$ & 0.095 \\
Transferrin $(\mathrm{g} / \mathrm{L})$ & $2.75(2.32,3.25)$ & $2.42(2.13,2.88)$ & 0.022 \\
Ferritin $(\mu \mathrm{gg} / \mathrm{L})$ & $124.0(43.0,306.0)$ & $354.0(189.5,865.5)$ & $<0.001$ \\
UIBC $(\mu \mathrm{mol} / \mathrm{L})$ & $39.3(23.3,51.6)$ & $25.0(16.2,32.9)$ & 0.005 \\
TIBC $(\mu \mathrm{mol} / \mathrm{L})$ & $64.4(56.1,72.4)$ & $55.9(51.7,61.5)$ & 0.004 \\
TSI $(\%)$ & $33.8(22.9,58.8)$ & $55.1(42.2,69.4)$ & 0.005 \\
\hline Data a express & &
\end{tabular}

Data are expressed as mean ( \pm standard deviation), median (quartile 25 , quartile 75) or number (proportion)

IgG immunoglobulin G, ALT alanine aminotransferase, AST aspartate aminotransferase, $A L P$ alkaline phosphatase, GGT gamma-glutamyl transpeptidase, TBIL total bilirubin, $A L B$ albumin, $P L T$ platelet, $P T$ prothrombin time, UIBC unsaturated iron binding capacity, TIBC Total iron binding capacity, $T S /$ transferrin saturation index

ALB and the PLT count $(P<0.05)$. TSI was positively correlated with PT $(P<0.05)$, while TIBC was negatively correlated with PT $(P<0.05)$.

\section{Analysis of risk factors associated with advanced liver fibrosis}

Binary logistic regression analysis was used to explore the risk factors associated with advanced liver fibrosis in AIH patients (Table 4). Patients with advanced liver fibrosis were older $(P=0.016)$; had higher serum IgG $(P=0.012)$, TBIL $(P=0.029)$, ferritin $(P=0.002)$, and TSI $(P=0.011)$; had longer PTs $(P<0.001)$ and lower PLT counts $(P<0.001)$; and had lower serum ALB $(P=0.039)$, transferrin $(P=0.043)$, UIBC $(P=0.008)$, and TIBC $(P=0.006)$ in the univariate logistic regression analysis. However, after the multivariate logistic regression analysis, the IgG, PLT count, PT, and ferritin 
Table 3 Correlations between iron metabolism related variables and clinical characteristics of patients

\begin{tabular}{|c|c|c|c|c|c|c|c|c|c|c|c|c|}
\hline \multirow[t]{2}{*}{ Variables statistics } & \multicolumn{2}{|c|}{ Iron $(\mu \mathrm{mol} / \mathrm{L})$} & \multicolumn{2}{|c|}{ Transferrin (g/L) } & \multicolumn{2}{|c|}{ Ferritin $(\mu \mathrm{g} / \mathrm{L})$} & \multicolumn{2}{|c|}{ UIBC ( $\mu \mathrm{mol} / \mathrm{L})$} & \multicolumn{2}{|c|}{$\mathrm{TIBC}(\mu \mathrm{mol} / \mathrm{L})$} & \multicolumn{2}{|l|}{ TSI (\%) } \\
\hline & $r$ & $P$ & $r$ & $P$ & $r$ & $P$ & $r$ & $P$ & $r$ & $P$ & $\mathbf{r}$ & $P$ \\
\hline Age (years) & 0.296 & 0.003 & -0.416 & $<0.001$ & 0.349 & $<0.001$ & -0.394 & $<0.001$ & -0.375 & $<0.001$ & 0.376 & $<0.001$ \\
\hline $\operatorname{lgG}(g / L)$ & 0.080 & 0.438 & 0.062 & 0.544 & 0.043 & 0.675 & -0.057 & 0.577 & 0.003 & 0.978 & 0.083 & 0.420 \\
\hline $\operatorname{ALT}(U / L)$ & 0.348 & $<0.001$ & -0.031 & 0.765 & 0.418 & $<0.001$ & -0.311 & 0.002 & -0.091 & 0.375 & 0.332 & 0.001 \\
\hline AST (U/L) & 0.462 & $<0.001$ & -0.097 & 0.346 & 0.460 & $<0.001$ & -0.415 & $<0.001$ & -0.107 & 0.299 & 0.453 & $<0.001$ \\
\hline ALP (U/L) & 0.310 & 0.002 & -0.029 & 0.776 & 0.209 & 0.040 & -0.251 & 0.013 & -0.080 & 0.437 & 0.299 & 0.003 \\
\hline GGT (U/L) & 0.408 & $<0.001$ & -0.104 & 0.312 & 0.340 & 0.001 & -0.350 & $<0.001$ & -0.136 & 0.186 & 0.390 & $<0.001$ \\
\hline TBIL $(\mu \mathrm{mol} / \mathrm{L})$ & 0.498 & $<0.001$ & -0.220 & 0.030 & 0.395 & $<0.001$ & -0.526 & $<0.001$ & -0.249 & 0.014 & 0.533 & $<0.001$ \\
\hline ALB $(g / L)$ & -0.392 & $<0.001$ & 0.344 & 0.001 & -0.271 & 0.007 & 0.533 & $<0.001$ & 0.405 & $<0.001$ & -0.515 & $<0.001$ \\
\hline PLT $\left(\times 10^{9} / \mathrm{L}\right)$ & -0.201 & 0.048 & 0.345 & 0.001 & -0.186 & 0.069 & 0.329 & 0.001 & 0.394 & $<0.001$ & -0.308 & 0.002 \\
\hline PT (s) & 0.154 & 0.132 & -0.176 & 0.085 & 0.159 & 0.119 & -0.207 & 0.042 & -0.224 & 0.028 & 0.235 & 0.021 \\
\hline Iron ( $\mu \mathrm{mol} / \mathrm{L})$ & NA & NA & -0.439 & $<0.001$ & 0.583 & $<0.001$ & -0.859 & $<0.001$ & -0.244 & 0.016 & 0.933 & $<0.001$ \\
\hline Transferrin (g/L) & -0.439 & $<0.001$ & NA & NA & -0.407 & $<0.001$ & 0.698 & $<0.001$ & 0.719 & $<0.001$ & -0.606 & $<0.001$ \\
\hline Ferritin $(\mu \mathrm{g} / \mathrm{L})$ & 0.583 & $<0.001$ & -0.407 & $<0.001$ & NA & NA & -0.604 & $<0.001$ & -0.328 & 0.001 & 0.623 & $<0.001$ \\
\hline UIBC ( $\mu \mathrm{mol} / \mathrm{L})$ & -0.859 & $<0.001$ & 0.698 & $<0.001$ & -0.604 & $<0.001$ & NA & NA & 0.652 & $<0.001$ & -0.965 & $<0.001$ \\
\hline TIBC ( $\mu \mathrm{mol} / \mathrm{L})$ & -0.244 & 0.016 & 0.719 & $<0.001$ & -0.328 & 0.001 & 0.652 & $<0.001$ & NA & NA & -0.486 & $<0.001$ \\
\hline TSI (\%) & 0.933 & $<0.001$ & -0.606 & $<0.001$ & 0.623 & $<0.001$ & -0.965 & $<0.001$ & -0.486 & $<0.001$ & NA & NA \\
\hline Fibrosis stage & 0.242 & 0.017 & -0.254 & 0.012 & 0.395 & $<0.001$ & -0.278 & 0.006 & -0.227 & 0.025 & 0.292 & 0.004 \\
\hline
\end{tabular}

NA not applicable

IgG immunoglobulin G, ALT alanine aminotransferase, AST aspartate aminotransferase, ALP alkaline phosphatase, GGT gamma-glutamyl transpeptidase, TBIL total bilirubin, ALB albumin, PLT platelet, PT prothrombin time, UIBC unsaturated iron binding capacity, TIBC Total iron binding capacity, TSI transferrin saturation index

Table 4 Univariate and multivariate analysis of factors associated with advanced liver fibrosis

\begin{tabular}{|c|c|c|c|c|}
\hline \multirow[t]{2}{*}{ Variables } & \multicolumn{2}{|l|}{ Univariate analysis } & \multicolumn{2}{|c|}{ Multivariate analysis } \\
\hline & OR $(95 \% \mathrm{Cl})$ & $P$ & OR $(95 \% \mathrm{Cl})$ & $P$ \\
\hline Age (years) & $1.053(1.010,1.097)$ & 0.016 & & \\
\hline Female & $0.960(0.312,2.955)$ & 0.943 & & \\
\hline Autoantibodies positive & $2.674(0.694,10.302)$ & 0.153 & & \\
\hline $\lg G(g / L)$ & $1.084(1.018,1.154)$ & 0.012 & $1.123(1.023,1.232)$ & 0.014 \\
\hline $\operatorname{ALT}(U / L)$ & $1.000(0.999,1.001)$ & 0.820 & & \\
\hline AST (U/L) & $1.001(0.999,1.002)$ & 0.314 & & \\
\hline $\mathrm{ALP}(\mathrm{U} / \mathrm{L})$ & $1.006(0.999,1.014)$ & 0.104 & & \\
\hline GGT (U/L) & $1.002(0.999,1.004)$ & 0.273 & & \\
\hline TBIL $(\mu \mathrm{mol} / \mathrm{L})$ & $1.006(1.001,1.012)$ & 0.029 & & \\
\hline $\operatorname{ALB}(g / L)$ & $0.921(0.851,0.996)$ & 0.039 & & \\
\hline $\operatorname{PLT}\left(\times 10^{9} / \mathrm{L}\right)$ & $0.984(0.976,0.992)$ & $<0.001$ & $0.988(0.979,0.997)$ & 0.013 \\
\hline PT (s) & $1.876(1.317,2.673)$ & $<0.001$ & $1.758(1.143,2.704)$ & 0.010 \\
\hline Iron ( $\mu \mathrm{mol} / \mathrm{L})$ & $1.023(0.994,1.054)$ & 0.125 & & \\
\hline Transferrin (g/L) & $0.494(0.250,0.977)$ & 0.043 & & \\
\hline Ferritin $(\mu \mathrm{g} / \mathrm{L})$ & $1.002(1.001,1.003)$ & 0.002 & $1.002(1.001,1.004)$ & 0.012 \\
\hline UIBC ( $\mu \mathrm{mol} / \mathrm{L})$ & $0.969(0.947,0.992)$ & 0.008 & & \\
\hline TIBC ( $\mu \mathrm{mol} / \mathrm{L})$ & $0.943(0.905,0.984)$ & 0.006 & & \\
\hline TSI (\%) & $1.023(1.005,1.041)$ & 0.011 & & \\
\hline
\end{tabular}

OR odds ratio, $C l$ confidence interval, I $g$ G immunoglobulin $G, A L T$ alanine aminotransferase, $A S T$ aspartate aminotransferase, $A L P$ alkaline phosphatase, GGT gammaglutamyl transpeptidase, TBIL total bilirubin, $A L B$ albumin, $P L T$ platelet, $P T$ prothrombin time, UIBC unsaturated iron binding capacity, $T I B C$ total iron binding capacity, TSI transferrin saturation index 


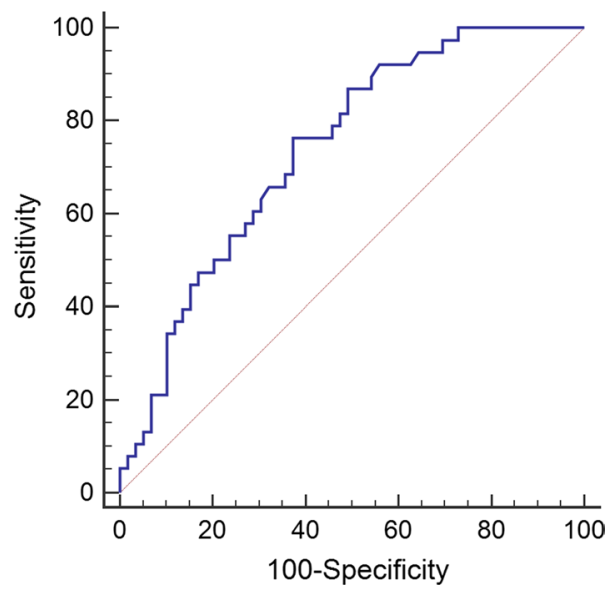

Fig. 1 Ferritin ROC curve analysis by the DeLong method. ROC curve of ferritin for the prediction of advanced liver fibrosis in AlH. ROC receiver operating characteristic, $A / H$ autoimmune hepatitis were independent risk factors for predicting advanced liver fibrosis in AIH patients, with odds ratios (ORs) (95\% confidence intervals [CIs]) of $1.123(1.023,1.232)$, $0.988(0.979,0.997), 1.758(1.143,2.704)$, and 1.002 (1.001, 1.004), respectively.

\section{Analysis of the diagnostic performance of ferritin using the ROC curve}

The ROC curve showed that the best ferritin cut-off value was $199 \mu \mathrm{g} / \mathrm{L}$, with $76.32 \%$ sensitivity and $62.71 \%$ specificity for the diagnosis of advanced liver fibrosis (area under the curve $[\mathrm{AUC}]=0.738,95 \%$ CI $0.639-0.822$; Fig. 1). Therefore, we compared the general characteristics of patients according to this cut-off level of ferritin. The age, ALT, AST, TBIL, iron, TSI, inflammatory grade, and fibrosis stage in the higher ferritin level group were significantly higher than those in the lower ferritin level group $(\mathrm{P}<0.05)$, while transferrin, UIBC, and TIBC in

Table 5 General characteristics of the patients by ferritin level

\begin{tabular}{|c|c|c|c|}
\hline Variables & Ferritin $\leq 199(\mu \mathrm{g} / \mathrm{L})(\mathrm{n}=46)$ & Ferritin $>199(\mu \mathrm{g} / \mathrm{L})(\mathrm{n}=51)$ & $P$ \\
\hline Age (years) & $49.6 \pm 11.7$ & $57.9 \pm 9.4$ & $<0.001$ \\
\hline Female, n (\%) & $41(89.1)$ & $41(80.4)$ & 0.235 \\
\hline Autoantibodies positive, n (\%) & $38(82.6)$ & $45(88.2)$ & 0.431 \\
\hline $\lg G(g / L)$ & $16.50(13.38,21.80)$ & $16.80(12.90,21.00)$ & 0.694 \\
\hline $\mathrm{ALT}(\mathrm{U} / \mathrm{L})$ & $50.0(23.0,152.8)$ & $124.0(44.0,482.0)$ & 0.006 \\
\hline AST (U/L) & $64.0(28.0,179.3)$ & $209.0(47.0,542.0)$ & 0.003 \\
\hline ALP (U/L) & $101.0(78.8,148.0)$ & $118.0(72.0,151.0)$ & 0.549 \\
\hline GGT (U/L) & $110.5(63.5,174.3)$ & $147.0(56.0,301.0)$ & 0.116 \\
\hline TBIL ( $\mu \mathrm{mol} / \mathrm{L})$ & $15.4(11.7,32.2)$ & $25.4(14.6,133.7)$ & 0.009 \\
\hline $\operatorname{ALB}(g / L)$ & $41.1 \pm 5.6$ & $39.3 \pm 5.5$ & 0.106 \\
\hline $\mathrm{PLT}\left(\times 10^{9} / \mathrm{L}\right)$ & $195.4 \pm 79.4$ & $175.3 \pm 60.5$ & 0.162 \\
\hline PT (s) & $12.9 \pm 1.3$ & $13.4 \pm 1.6$ & 0.09 \\
\hline Iron $(\mu \mathrm{mol} / \mathrm{L})$ & $21.0 \pm 12.1$ & $33.7 \pm 13.7$ & $<0.001$ \\
\hline Transferrin (g/L) & $3.04(2.41,3.40)$ & $2.41(2.13,2.88)$ & 0.001 \\
\hline UIBC ( $\mu \mathrm{mol} / \mathrm{L})$ & $45.0(30.2,58.6)$ & $24.6(12.5,32.0)$ & $<0.001$ \\
\hline TIBC ( $\mu \mathrm{mol} / \mathrm{L})$ & $64.9(54.9,75.4)$ & $56.6(53.6,66.9)$ & 0.022 \\
\hline TSI (\%) & $30.9(19.1,50.2)$ & $56.9(43.3,80.8)$ & $<0.001$ \\
\hline Histology (inflammation), n (\%) & & & $<0.001$ \\
\hline G1 & $2(4.3)$ & $1(2.0)$ & \\
\hline G2 & $30(65.2)$ & $12(23.5)$ & \\
\hline G3 & $12(26.1)$ & $28(54.9)$ & \\
\hline G4 & $2(4.3)$ & 10 (19.6) & \\
\hline Histology (fibrosis), n (\%) & & & 0.001 \\
\hline F0 & $10(21.7)$ & $1(2.0)$ & \\
\hline F1 & $13(28.3)$ & $9(17.6)$ & \\
\hline $\mathrm{F} 2$ & $14(30.4)$ & $12(23.5)$ & \\
\hline F3 & $5(10.9)$ & $16(31.4)$ & \\
\hline F4 & $4(8.7)$ & $13(25.5)$ & \\
\hline
\end{tabular}

Data are expressed as mean ( \pm standard deviation), median (quartile 25 , quartile 75 ) or number (proportion)

IgG immunoglobulin G, ALT alanine aminotransferase, AST aspartate aminotransferase, ALP alkaline phosphatase, GGT gamma-glutamyl transpeptidase, TBIL total bilirubin, $A L B$ albumin, PLT platelet, PT prothrombin time, UIBC unsaturated iron binding capacity, TIBC total iron binding capacity, TSI transferrin saturation index 
the higher ferritin level group were significantly lower than those in the lower ferritin level group $(P<0.05)$. Significant differences between the two groups were not observed for other parameters $(\mathrm{P}>0.05)$, as shown in Table 5 .

\section{Discussion}

To the best of our knowledge, the association between iron-metabolism-related variables and liver fibrosis has not been extensively studied in AIH patients. In this study, we found that higher serum ferritin levels were independently associated with advanced liver fibrosis in patients with treatment-naive autoimmune hepatitis. $\mathrm{AIH}$ is an immune-mediated chronic, progressive inflammatory liver disease with diverse clinical manifestations. Liver fibrosis is a common complication in the progression of $\mathrm{AIH}$, and many patients have advanced liver fibrosis at presentation [32]. Hence, the determination of liver fibrosis is also crucial for the determination of the prognosis of $\mathrm{AIH}$ and for the selection of treatment. These findings reveal the influence of ferritin on hepatic fibrosis in AIH patients. Serum ferritin is a noninvasive indicator that can be easily evaluated in clinical laboratories. Therefore, serum ferritin can be used as a routine reference index for monitoring hepatic fibrosis in all $\mathrm{AIH}$ patients.

Iron is derived from dietary sources containing haem $(\leq 10 \%)$ and nonheme $(>90 \%)$ products $[33,34]$. Extracellular ferrous iron is oxidized to ferric iron by oxidizing ferroxidase (hephaestin), and inactive ferric iron is bound to transferrin [35]. Transferrin is a protein synthesized and released by the liver and is responsible for iron transport through the plasma. In general, transferrin is increased in absolute iron deficiency to maximize the use of the limited available iron [36]. However, during inflammation, the ability of the liver to synthesize proteins, including transferrin, is reduced. UIBC and TIBC can be used as alternative tests for transferrin [37]. Similarly, we found that serum transferrin levels were positively correlated with UIBC and TIBC. As part of a complex system that senses plasma iron levels, transferrin can also regulate the activity of hepcidin [38]. Hepcidin is mostly produced by hepatocytes in response to iron load in cells. Ferritin is the main storage protein for iron, and the liver is the main ferritin storage site [39]. Moreover, ferritin is an acute phase protein that can be increased in the process of inflammation, whereas transferrin is downregulated in the process of inflammation and reduced in advanced liver disease [40-42], which is in accordance with our results. In addition, TSI is essential for identifying hepatic iron overload, and Ribot et al. reported that TSI was related to liver function damage and inflammation [43], which was partially consistent with our results.
Disturbances in iron regulation have been reported in diverse chronic liver diseases other than hereditary haemochromatosis [16-20]. Serum ferritin levels are increased in patients with chronic hepatitis B [44], chronic hepatitis $C$ [45-47], chronic alcoholic liver disease [48], nonalcoholic fatty liver disease [25, 26, 48], and AIH [21, 22]. Disturbances in iron homeostasis in chronic liver diseases may be a manifestation of active inflammation and parenchymal damage [18, 40, 49] or a pathological mechanism by which iron toxicity aggravates liver injury and outcome $[39,50]$. Iron overload can lead to oxidative stress, which has been implicated in the pathogenesis of many chronic liver diseases [51-54]. The production of reactive oxygen species can impair the cell membrane, damage mitochondrial function, affect gene expression, induce hepatocyte injury, and promote liver fibrosis [55].

Serum ferritin levels and serum iron concentrations were found to be increased in $65 \%$ and $58 \%$ of patients with untreated AIH [21]. Serum ferritin levels paralleled serum aminotransferase levels in patients with $\mathrm{AIH}$ and chronic hepatitis $C[21,56]$, which was consistent with our study's findings. The iron disturbances in untreated AIH may reflect increased iron release from damaged hepatocytes, and their subsequent resolution with treatment-induced normalization of aminotransferase and IgG reflects their remediation after successful treatment $[21,40]$. The increase in liver iron promotes the synthesis of ferritin [57], which can activate the production of collagen and liver fibrogenesis [58]. More specifically, ferritin can activate hepatic stellate cells, which are key effectors of fibrogenesis. Although several prior studies have reported significant relationships between ferritin levels and liver fibrosis [24, 58-60], others have reported disparate findings $[46,60]$. Therefore, we conducted this study to assess whether serum ferritin (and/or other ironmetabolism-related variables) was related to advanced liver fibrosis in $\mathrm{AIH}$ patients.

We also demonstrated that a higher serum IgG, lower PLT count, and longer PT were independent risk factors to predict advanced liver fibrosis in AIH patients. Our findings share many similarities with findings from several prior publications $[4,61-64]$. Therefore, we believe that patients with higher serum ferritin, higher serum IgG, a lower PLT count, and a longer PT are more likely to have advanced liver fibrosis.

This study has a few limitations. First, the number of patients is relatively small because of the low prevalence of AIH. Sample size determination through calculations and statistical power determination are important in the validation of study findings. However, we did not formally determine our sample size through calculations, as our sample size was determined by the availability of existing data. 
Second, because this was a cross-sectional study, we could not demonstrate a causal link between ferritin and the risk of advanced liver fibrosis. In addition, the retrospective nature of the study raises the possibility of bias. Despite these limitations, our study investigated the relationship between ferritin levels and advanced liver fibrosis in $\mathrm{AIH}$ patients to determine whether serum ferritin could be used as a routine reference index for monitoring hepatic fibrosis in $\mathrm{AIH}$ patients. Further prospective large-scale studies are needed to confirm the causal relationship.

In summary, we showed that higher serum ferritin was independently associated with advanced liver fibrosis among patients with treatment-naive $\mathrm{AIH}$, especially if combined with thrombocytopenia, a prolonged PT, and increased IgG. Serum ferritin may be used to predict the presence of advanced liver fibrosis in the clinic, but additional investigations are warranted for validation.

\author{
Abbreviations \\ AlH: Autoimmune hepatitis; IgG: Immunoglobulin G; ALT: Alanine aminotrans- \\ ferase; AST: Aspartate aminotransferase; ALP: Alkaline phosphatase; GGT \\ : Gamma-glutamyl transpeptidase; TBIL: Total bilirubin; ALB: Albumin; UIBC: \\ Unsaturated iron binding capacity; TIBC: Total iron binding capacity; TSI: \\ Transferrin saturation index; PT: Prothrombin time; PLT: Platelet; OR: Odds ratio; \\ $\mathrm{Cl}$ : Confidence interval.
}

\section{Acknowledgements}

We thank the medical staff at Tianjin Second People's Hospital for their essential support and assistance with data collection.

\section{Authors' contributions \\ Study concept and design (QC, JL), drafting manuscript (QC, MG), statistical analysis $(H Y, R Z, P L)$, data collection (HY, LM, LZ, PH, JW), and the revision of the manuscript for important intellectual content $(M G, J L)$. All authors read and approved the final manuscript.}

\section{Funding}

No funding was provided for this study.

\section{Availability of data and materials}

The datasets used and/or analysed during the current study are available from the corresponding author upon reasonable request.

\section{Declarations}

\section{Ethics approval and consent to participate}

This study protocol was approved by the Ethics Committee of Tianjin Second People's Hospital and conducted according to the principles of the Declaration of Helsinki. We obtained written informed consent from all patients.

\section{Consent for publication}

Not applicable.

\section{Competing interests}

All authors declare no competing interest.

\section{Author details}

'Department of Hepatology, Second People's Clinical College of Tianjin Medical University, Tianjin, China. ${ }^{2}$ Department of Hepatology, Tianjin Second People's Hospital, No. 7, Sudi South Road, Nankai District, Tianjin 300192, China. ${ }^{3}$ Department of Neurology, The First Hospital of Jilin University, Changchun, Jilin, China.
Received: 24 September 2021 Accepted: 28 December 2021

Published online: 17 January 2022

\section{References}

1. Manns MP, Lohse AW, Vergani D. Autoimmune hepatitis-update 2015. J Hepatol. 2015;62(1 Suppl):S100-11.

2. Czaja AJ. Autoimmune hepatitis in diverse ethnic populations and geographical regions. Expert Rev Gastroenterol Hepatol. 2013;7(4):365-85.

3. Yang F, Wang Q, Bian Z, Ren LL, Jia J, Ma X. Autoimmune hepatitis: east meets west. J Gastroenterol Hepatol. 2015;30(8):1230-6.

4. Wang QX, Yan L, Ma X. Autoimmune hepatitis in the Asia-Pacific Area. J Clin Transl Hepatol. 2018;6(1):48-56.

5. Sahebjam F, Vierling JM. Autoimmune hepatitis. Front Med. 2015;9(2):187-219.

6. Manns MP, Czaja AJ, Gorham JD, Krawitt EL, Mieli-Vergani G, Vergani D, Vierling JM. Diagnosis and management of autoimmune hepatitis. Hepatology. 2010;51(6):2193-213.

7. EASL Clinical Practice Guidelines. Autoimmune hepatitis. J Hepatol. 2015;63(4):971-1004.

8. Regev A, Berho M, Jeffers LJ, Milikowski C, Molina EG, Pyrsopoulos NT, Feng ZZ, Reddy KR, Schiff ER. Sampling error and intraobserver variation in liver biopsy in patients with chronic HCV infection. AM J Gastroenterol. 2002;97(10):2614-8.

9. Mehta SH, Lau B, Afdhal NH, Thomas DL. Exceeding the limits of liver histology markers. J Hepatol. 2009;50(1):36-41.

10. Kim WR, Berg T, Asselah T, Flisiak R, Fung S, Gordon SC, Janssen HL, Lampertico P, Lau D, Bornstein JD, et al. Evaluation of APRI and FIB-4 scoring systems for non-invasive assessment of hepatic fibrosis in chronic hepatitis B patients. J Hepatol. 2016;64(4):773-80.

11 Vallet-Pichard A, Mallet V, Nalpas B, Verkarre V, Nalpas A, Dhalluin-Venier $\checkmark$, Fontaine H, Pol S. FIB-4: an inexpensive and accurate marker of fibrosis in HCV infection. Comparison with liver biopsy and fibrotest. Hepatology. 2007:46(1):32-6.

12. Castera L. Diagnosis of non-alcoholic fatty liver disease/non-alcoholic steatohepatitis: non-invasive tests are enough. Liver int. 2018;38(Suppl 1):67-70.

13. Herrmann E, de Ledinghen V, Cassinotto C, Chu WC, Leung VY, Ferraioli G, Filice C, Castera L, Vilgrain V, Ronot M, et al. Assessment of biopsy-proven liver fibrosis by two-dimensional shear wave elastography: an individual patient data-based meta-analysis. Hepatology. 2018;67(1):260-72.

14. Hartl J, Denzer U, Ehlken H, Zenouzi R, Peiseler M, Sebode M, Hubener S, Pannicke N, Weiler-Normann C, Quaas A, et al. Transient elastography in autoimmune hepatitis: timing determines the impact of inflammation and fibrosis. J Hepatol. 2016;65(4):769-75.

15. Datz C, Felder TK, Niederseer D, Aigner E. Iron homeostasis in the metabolic syndrome. Eur J Clin Invest. 2013;43(2):215-24.

16. Alla V, Bonkovsky HL. Iron in nonhemochromatotic liver disorders. Semin Liver Dis. 2005;25(4):461-72.

17. Hearnshaw S, Thompson NP, McGill A. The epidemiology of hyperferritinaemia. World J Gastroenterol. 2006;12(36):5866-9.

18. Camaschella C, Poggiali E. Towards explaining "unexplained hyperferritinemia." Haematologica. 2009;94(3):307-9.

19. Fargion S, Valenti L, Fracanzani AL. Beyond hereditary hemochromatosis: new insights into the relationship between iron overload and chronic liver diseases. Dig Liver Dis. 2011;43(2):89-95.

20. Siddique A, Kowdley KV. Review article: the iron overload syndromes. Aliment Pharmacol Ther. 2012;35(8):876-93.

21. Taubert R, Hardtke-Wolenski M, Noyan F, Lalanne C, Jonigk D, Schlue J, Krech T, Lichtinghagen R, Falk CS, Schlaphoff $\mathrm{V}$, et al. Hyperferritinemia and hypergammaglobulinemia predict the treatment response to standard therapy in autoimmune hepatitis. PLOS ONE. 2017;12(6):e179074.

22. An IC, Tiwari AK, Ameda S, Laird-Fick HS. Autoimmune hepatitis: diagnostic dilemma in the setting of suspected iron overload. Case Rep Gastrointest Med. 2013;2013:872987.

23. Lange CM, Kutalik Z, Morikawa K, Bibert S, Cerny A, Dollenmaier G, Dufour JF, Gerlach TJ, Heim MH, Malinverni R, et al. Serum ferritin levels are associated with a distinct phenotype of chronic hepatitis $C$ poorly responding to pegylated interferon-alpha and ribavirin therapy. Hepatology. 2012;55(4):1038-47. 
24. Batsaikhan B, Gantumur G, Huang Cl, Yeh ML, Huang CF, Lin ZY, Chen SC, Huang JF, Yu ML, Chuang WL, et al. Elevated serum ferritin level associated with hepatic steatosis and fibrosis in hepatitis $C$ virus-infected patients. J Chin Med Assoc. 2019;82(2):99-104.

25. Manousou P, Kalambokis G, Grillo F, Watkins J, Xirouchakis E, Pleguezuelo M, Leandro G, Arvaniti V, Germani G, Patch D, et al. Serum ferritin is a discriminant marker for both fibrosis and inflammation in histologically proven non-alcoholic fatty liver disease patients. Liver Int. 2011;31(5):730-9.

26. Kowdley KV, Belt P, Wilson LA, Yeh MM, Neuschwander-Tetri BA, Chalasani $\mathrm{N}$, Sanyal AJ, Nelson JE. Serum ferritin is an independent predictor of histologic severity and advanced fibrosis in patients with nonalcoholic fatty liver disease. Hepatology. 2012;55(1):77-85.

27. Jung JY, Shim JJ, Park SK, Ryoo JH, Choi JM, Oh IH, Jung KW, Cho H, Ki $\mathrm{M}$, Won $\mathrm{YJ}$, et al. Serum ferritin level is associated with liver steatosis and fibrosis in Korean general population. Hepatol Int. 2019;13(2):222-33.

28. Alvarez F, Berg PA, Bianchi FB, Bianchi L, Burroughs AK, Cancado EL, Chapman RW, Cooksley WG, Czaja AJ, Desmet VJ, et al. International Autoimmune Hepatitis Group Report: review of criteria for diagnosis of autoimmune hepatitis. J Hepatol. 1999:31(5):929-38.

29. Hennes EM, Zeniya M, Czaja AJ, Pares A, Dalekos GN, Krawitt EL, Bittencourt PL, Porta G, Boberg KM, Hofer H, et al. Simplified criteria for the diagnosis of autoimmune hepatitis. Hepatology. 2008;48(1):169-76.

30. Goodman ZD. Grading and staging systems for inflammation and fibrosis in chronic liver diseases. J Hepatol. 2007:47(4):598-607.

31 Batts KP, Ludwig J. Chronic hepatitis. An update on terminology and reporting. Am J Surg Pathol. 1995:19(12):1409-17.

32. Mieli-Vergani G, Vergani D. Autoimmune hepatitis. Nat Rev Gastroenterol Hepatol. 2011;8(6):320-9.

33. Siah CW, Ombiga J, Adams LA, Trinder D, Olynyk JK. Normal iron metabolism and the pathophysiology of iron overload disorders. Clin Biochem Rev. 2006;27(1):5-16.

34. Milto IV, Suhodolo IV, Prokopieva VD, Klimenteva TK. Molecular and cellular bases of iron metabolism in humans. Biochemistry. 2016;81(6):549-64.

35. Vulpe CD, Kuo YM, Murphy TL, Cowley L, Askwith C, Libina N, Gitschier

J, Anderson GJ. Hephaestin, a ceruloplasmin homologue implicated in intestinal iron transport, is defective in the sla mouse. Nat Genet. 1999:21(2):195-9.

36. Adams PC, Barton JC. A diagnostic approach to hyperferritinemia with a non-elevated transferrin saturation. J Hepatol. 2011;55(2):453-8.

37. Fairbanks VF. Laboratory testing for iron status. Hosp Pract. 1991;26(Suppl 3):17-24.

38. Gkouvatsos K, Papanikolaou G, Pantopoulos K. Regulation of iron transport and the role of transferrin. Biochim Biophys Acta. 2012;1820(3):188-202.

39. Harrison PM, Arosio P. The ferritins: molecular properties, iron storage function and cellular regulation. Biochim Biophys Acta. 1996;1275(3):161-203.

40. Bhagat $\mathrm{Cl}$, Fletcher S, Joseph J, Beilby JP. Plasma ferritin in acute hepatocellular damage. Clin Chem. 2000;46(6 Pt 1):885-6.

41. Ritchie RF, Palomaki GE, Neveux LM, Navolotskaia O, Ledue TB, Craig WY. Reference distributions for immunoglobulins $A, G$, and $M$ : a practical, simple, and clinically relevant approach in a large cohort. J Clin Lab Anal. 1998;12(6):363-70.

42. Bruns T, Nuraldeen R, Mai M, Stengel S, Zimmermann HW, Yagmur E, Trautwein C, Stallmach A, Strnad P. Low serum transferrin correlates with acute-on-chronic organ failure and indicates short-term mortality in decompensated cirrhosis. Liver Int. 2017;37(2):232-41.

43. Ribot-Hernandez I, Martin-Gonzalez C, Vera-Delgado V, GonzalezNavarrete L, de Armas-Gonzalez JF, Vina-Rodriguez J, Sanchez-Perez MJ, Rodriguez-Gaspar M, Gonzalez-Reimers E. Prognostic value of serum iron, ferritin, and transferrin in chronic alcoholic liver disease. Biol Trace Elem Res. 2020;195(2):427-35

44. Sebastiani G, Tempesta D, Alberti A. Hepatic iron overload is common in chronic hepatitis $B$ and is more severe in patients coinfected with hepatitis D virus. J Viral Hepat. 2012;19(2):e170-6.

45. Bonkovsky HL. Iron as a comorbid factor in chronic viral hepatitis. Am J Gastroenterol. 2002;97(1):1-4.

46. D'Souza RF, Feakins R, Mears L, Sabin CA, Foster GR. Relationship between serum ferritin, hepatic iron staining, diabetes mellitus and fibrosis progression in patients with chronic hepatitis C. Aliment Pharmacol Ther. 2005;21(5):519-24.

47. Valenti L, Pulixi EA, Arosio P, Cremonesi L, Biasiotto G, Dongiovanni P, Maggioni M, Fargion S, Fracanzani AL. Relative contribution of iron genes, dysmetabolism and hepatitis C virus (HCV) in the pathogenesis of altered iron regulation in HCV chronic hepatitis. Haematologica. 2007:92(8):1037-42.

48. Bell H, Skinningsrud A, Raknerud N, Try K. Serum ferritin and transferrin saturation in patients with chronic alcoholic and non-alcoholic liver diseases. J Intern Med. 1994;236(3):315-22

49. Bugianesi E, Manzini P, D'Antico S, Vanni E, Longo F, Leone N, Massarenti P, Piga A, Marchesini G, Rizzetto M. Relative contribution of iron burden, HFE mutations, and insulin resistance to fibrosis in nonalcoholic fatty liver. Hepatology. 2004;39(1):179-87.

50. Ganz T. Systemic iron homeostasis. Physiol Rev. 2013;93(4):1721-41.

51. Koek GH, Liedorp PR, Bast A. The role of oxidative stress in non-alcoholic steatohepatitis. Clin Chim Acta. 2011;412(15-16):1297-305.

52. Galicia-Moreno M, Gutierrez-Reyes $\mathrm{G}$. The role of oxidative stress in the development of alcoholic liver disease. Rev Gastroenterol Mex. 2014;79(2):135-44.

53. Pemberton PW, Aboutwerat A, Smith A, Burrows PC, McMahon RF, Warnes TW. Oxidant stress in type I autoimmune hepatitis: the link between necroinflammation and fibrogenesis? Biochim Biophys Acta. 2004;1689(3):182-9.

$54 \mathrm{Choi} \mathrm{J,} \mathrm{Ou} \mathrm{JH.} \mathrm{Mechanisms} \mathrm{of} \mathrm{liver} \mathrm{injury.} \mathrm{III.} \mathrm{Oxidative} \mathrm{stress} \mathrm{in} \mathrm{the}$ pathogenesis of hepatitis C virus. Am J Physiol Gastrointest Liver Physiol. 2006:290(5):G847-51.

55. Czaja AJ. Nature and implications of oxidative and nitrosative stresses in autoimmune hepatitis. Dig Dis Sci. 2016;61(10):2784-803.

56. Shan Y, Lambrecht RW, Bonkovsky HL. Association of hepatitis C virus infection with serum iron status: analysis of data from the third National Health and Nutrition Examination Survey. Clin Infect Dis. 2005;40(6):834-41.

57. Tran TN, Eubanks SK, Schaffer KJ, Zhou CY, Linder MC. Secretion of ferritin by rat hepatoma cells and its regulation by inflammatory cytokines and iron. Blood. 1997;90(12):4979-86.

58. Wang W, Knovich MA, Coffman LG, Torti FM, Torti SV. Serum ferritin: past, present and future. Biochim Biophys Acta. 2010;1800(8):760-9.

59. Afify SM, Tabll A, Nawara HM, El KM, Elfert A, Seno M, El-Kousy S. Five fibrosis biomarkers together with serum ferritin level to diagnose liver fibrosis and cirrhosis. Clin Lab. 2018;64(10):1685-93.

60. Costa-Matos L, Batista P, Monteiro N, Simoes M, Egas C, Pereira J, Pinho H, Santos N, Ribeiro J, Cipriano MA, et al. Liver hepcidin mRNA expression is inappropriately low in alcoholic patients compared with healthy controls. Eur J Gastroenterol Hepatol. 2012;24(10):1158-65.

61. Martin-Gonzalez C, Pelazas-Gonzalez R, Fernandez-Rodriquez C, AlemanValls R, Martinez-Riera A, Ortega-Toledo P, Garcia-Rodriguez A, Rodriguez-Gaspar M, Gonzalez-Reimers E. Ferritin and liver fibrosis among patients with chronic hepatitis C virus infection. J Trace Elem Med Biol. 2020;61:126542.

62. Puustinen L, Boyd S, Mustonen H, Arkkila P, Arola J, Farkkila M. Prognostic value of clinical variables and liver histology for development of fibrosis and cirrhosis in autoimmune hepatitis. Scand J Gastroenterol. 2017;52(3):321-7

63. Liu L, Cao J, Zhong Z, Guo Z, Jiang Y, Bai Y, Xu J. Noninvasive indicators predict advanced liver fibrosis in autoimmune hepatitis patients. J Clin Lab Anal. 2019;33(7):e22922.

64. Yuan X, Duan SZ, Cao J, Gao N, Xu J, Zhang L. Noninvasive inflammatory markers for assessing liver fibrosis stage in autoimmune hepatitis patients. Eur J Gastroenterol Hepatol. 2019:31(11):1467-74.

\section{Publisher's Note}

Springer Nature remains neutral with regard to jurisdictional claims in published maps and institutional affiliations. 